\title{
Bioinspired synthesis of optically and thermally responsive nanoporous membranes
}

\author{
J Rubén Morones-Ramírez
}

The development of porous membranes that can rapidly change flow rates in response to external, noninvasive stimuli has broad technological applications for areas ranging from biomedical devices to architecture. Environmentally responsive membranes have a fundamental role in the development of devices used in chemical sensors, biological sorters, sequencing, separations, high-throughput medical devices and labs on a chip. The design and engineering of these responsive porous membranes have been achieved through the coupling of porous membranes with polymeric materials that can change their physical conformation in response to pressure, heat, $\mathrm{pH}$ or different chemical entities. Inspired by the phototropic growth of coleoptiles and the light-mediated mechanism that plants use to open their stomata, in this work, light-responsive porous membranes were engineered, mathematically modeled and synthesized. This biologically inspired approach led to a state-of-the-art design technique and a device that outperforms its natural counterpart and is capable of reversibly controlling flow rates from 0.001 to $0.035 \mathrm{ml} \mathrm{s}^{-1} \mathrm{~cm}^{-2}$ in less than a few minutes using the noninvasive stimulus of light. We envision that the polymeric responsive membranes and the platform synthesis technique employed in this manuscript for their fabrication could be utilized in a broad range of applications and will have a great impact on the fields of fluid handling, biomedical high-throughput devices, sensors, medicine and other fields of chemistry, biology and mechanical engineering.

NPG Asia Materials (2013) 5, e52; doi:10.1038/am.2013.23; published online 21 June 2013

Keywords: intelligent responsive polymers; nanocomposites; optically responsive materials; porous membranes; thermally responsive materials

\section{INTRODUCTION}

Development of nanoporous membranes capable of changing their permeability in response to changes in environmental stimuli is a very appealing area of research. ${ }^{1-3}$ Environmentally responsive porous membranes (ERPMs) have a wide array of applications, including use in sensors, bioseparation, drug delivery systems and valves that serve to interconnect microfluidic systems and control interflow. ${ }^{3-12}$ For decades, the efforts to develop ERPMs have been approached mainly through the coupling of environmentally responsive polymers to polymeric porous membranes. This technique allows for rapid 'on/ off' switching and flow control because it synergizes the chemical stability and mechanical strength of the polymer membrane with the fast response times of the free-ended polymer chains chemically bonded to them. As a result, different composite membranes, capable of changing their effective pore size with environmental triggers, such as $\mathrm{pH}$ and ionic strength, ${ }^{13,14}$ temperature, ${ }^{15}$ chemical moieties, such as glucose, ${ }^{16}$ and more recently, light, ${ }^{17-19}$ have been developed.

Despite decades of intense research in the field, fabrication of ERPMs has proven to be extremely challenging. Environmentally responsive membranes are still plagued with problems that restrict their practical applications. One of the most challenging problems is the engineering of a system that allows for fast response times in flow that can be controlled through an external stimulus and that does not require changing the environmental conditions of the entire system.

In this work, the inspiration to engineer and design an ERPM capable of rapidly controlling flow rates using a noninvasive stimulus (light) came from nature. In natural systems, conversion of light into another form of energy is done through photoreceptors or chlorophores. Such is the case of the phototropic growth of coleoptiles plants, ${ }^{20}$ where light is converted into a chemical response or the 'on/ off' switching of stomata in plants driven by light exposure (Scheme 1a). ${ }^{21}$ The system presented in this work was designed to include an opto-mechanical energy converter capable of controlling flow by mechanically switching the pores in the ERPM from 'on' to 'off' in response to light (Scheme 1b). For this purpose, a chlorophore synthetic analog was engineered through the chemical synthesis of a composite material that coupled temperature-responsive biopolymers with metallic nanoparticles (Scheme 1c). Metallic nanoparticles are excellent light-heat converters, and when coupled to a polymeric material that rapidly undergoes changes in its physical conformation due to heat, the perfect gate to incorporate into the nanopores is obtained (Schemes $1 \mathrm{a}$ and b). The system design, shown in Scheme $1 \mathrm{~b}$, overcame the challenges of slow response times and the 

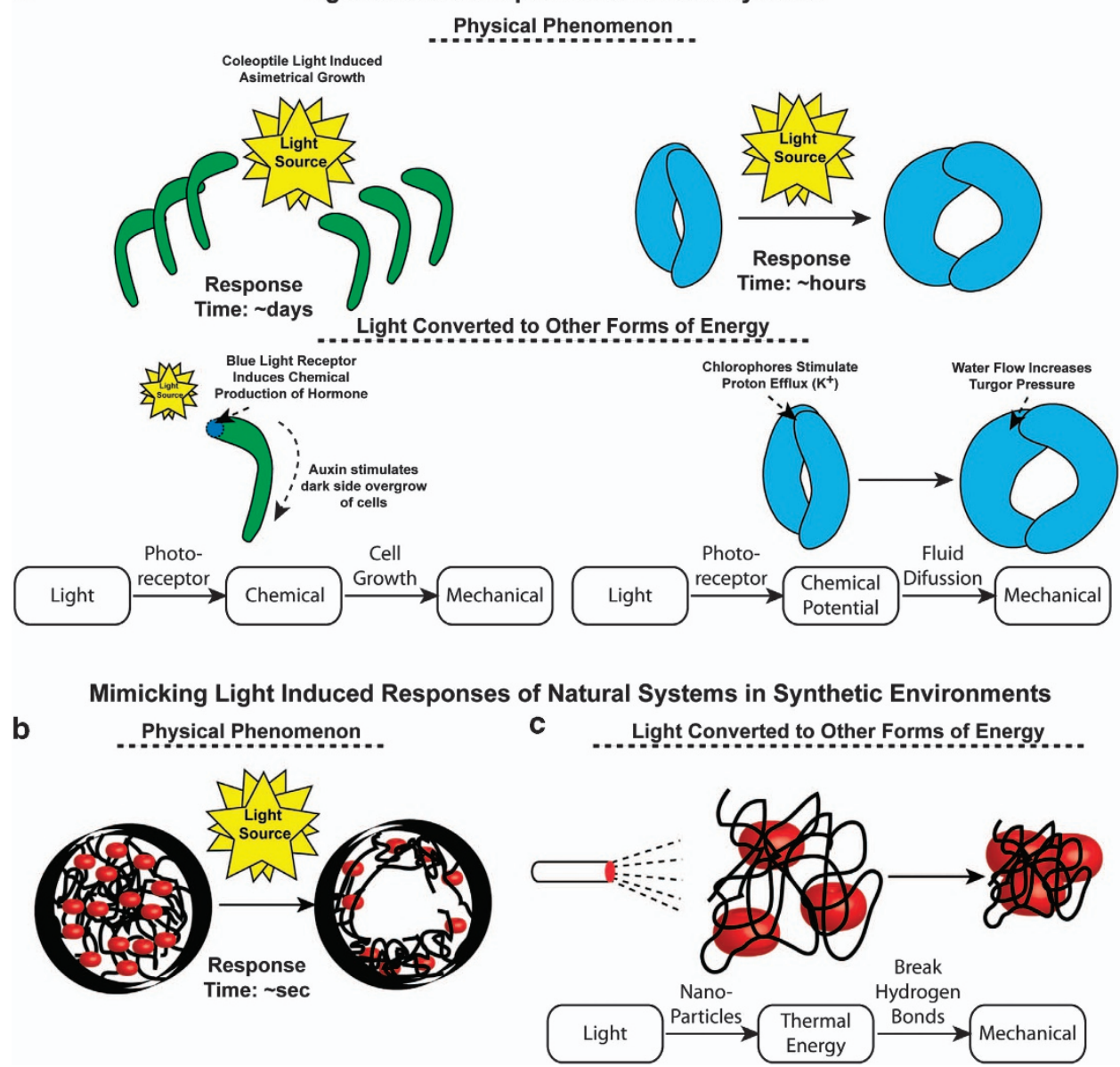

Scheme 1 Light-induced responses in natural systems. (a) Left panel shows a schematic of the mechanism of phototropic growth of coleoptiles. The photoresponse of this system is in the order of days. The energy conversion that takes place in this phenomenon is as follows: photoreceptors stimulate a chemical response in the form of a growth hormone, which leads to a mechanical stimulus in the form of growth in a specific direction. Right panel shows a schematic of the photoinduced mechanism by which stomata in plants are opened and closed. The photo-response of this system is in the order of hours The energy conversion that takes place in this phenomenon is as follows: chromophores stimulate a chemical response, in the form of a chemical potential, which leads to a mechanical stimulus, in the form of increased turgor pressure that allows opening the stomata. (b and $\mathbf{c}$ ) Design synthetic photo-responsive system. (b) Schematic of the systems, which illustrates how nanopores within porous membranes can be switched from 'on' to 'off' in response to a light stimulus. The response time of the system is in the order of seconds. (c) The switching mechanism is demonstrated as well as the energy conversion that takes place in the system, as follows: metallic nanoparticles convert light into localized heating, and the heating then stimulates a mechanical response, in the form of a physical conformation change in a thermo-responsive, polymer. This conformational change finally allows the closing or opening of pores.

requirement to change bulk system conditions to achieve flow control.

This manuscript reports the design, mathematical model and fabrication synthesis of a reversible fast-responding ERPM that uses light at specific wavelengths to control flow rates. This work proposes the development of a synthesis pathway to achieve grafting of opto-thermally responsive polymer-nanoparticle nanocomposites to polycarbonate track-etched porous membranes (PCTEPMs). The synthesis involves prior grafting of poly $(N$-isopropyl acrylamide $)$ (PNIPAM) chains to the walls of the polycarbonate membrane, followed by an in situ reduction of a metallic salt, $\mathrm{HAuCl}_{4}$. The synthesis pathway proposed is based on our previous work, ${ }^{11,22,23}$ which describes the capability of PNIPAM to be coupled to metallic particles, control their sizes and shapes, and form opto-thermally responsive nanocomposite materials. ${ }^{22,23}$

We present a bioinspired state-of-the-art technique and device that utilizes simplified mechanisms to outperform the 'on/off' switching of its natural counterpart. The 'on/off' switching is reversible and much faster than other methods, taking only seconds to respond as the mathematical modeling of the system predicts. The device also has better control compared with the photoreceptors in coleoptiles (which have a response time in the order of hours $)^{20}$ and the chlorophores in plants (which have a response time in the order of minutes) $)^{21}$ (Scheme 1). Therefore, we envision that these optically responsive porous membranes will be used in fluid handling, biomedical highthroughput devices, sensors, medicine and other fields of chemistry, biology and mechanical engineering.

\section{MATERIALS AND METHODS}

\section{Materials}

Deionized water with a resistivity of at least $18.0 \mathrm{M} \Omega \mathrm{cm}$ (EPure, Barnstead Thermolyne, Dubuque, IA, USA) was used in all experiments. N-Isopropyl acrylamide (NIPAM) (Fisher Scientific, Pittsburgh, PA, USA) was recrystallized once in hexane (Fisher Scientific) and stored at $-20^{\circ} \mathrm{C}$ until use. Ascorbic acid (AsA) (Fisher Scientific) and hydrogen tetrachloroaureate (III) hydrate $\left(\mathrm{HAuCl}_{4}\right)$ (Sigma Aldrich, St Louis, MO, USA) were used as purchased. 
PCTEPMs with a 400-nm diameter were obtained from Whatman (St Louis, MO, USA) and used as purchased.

Surface grafting of PNIPAM on pores of PCTE porous membranes using plasma-graft pore-filling surface polymerization

Linear PNIPAM chains were grafted into the pores of PCTEPMs of $400 \mathrm{~nm}$ diameter through a plasma-induced grafting polymerization technique reported by $\mathrm{Qu}^{24}$ The reaction involves placing the membranes in a transparent glass tube filled with air without any flow and then evacuating the tube at $350 \mu$ Torr. The substrate is then treated with a steady cloud of air plasma at $30 \mathrm{~W}$ for $60 \mathrm{~s}$. Immediately after treatment, the membrane is immersed in ethanol for $2 \mathrm{~s}$, and subsequently the membrane is submerged in a $5 \mathrm{wt} \%$ monomer (NIPAM) aqueous solution and left to polymerize for $3 \mathrm{~h}$. In the present study, the reaction was performed at both 20 and $30^{\circ} \mathrm{C}$. Following the reaction, the membrane was rinsed three times in deionized water, and then it was left in $50 \mathrm{ml}$ of deionized water for $24 \mathrm{~h}$ to remove any unreacted monomer.

\section{In situ synthesis of gold nanoparticles on PNIPAM grafts}

We grew gold nanoparticles in the pores by the following steps: PCTEPMs with surface-grafted PNIPAM (gPCETPMs) were submerged into a 5-ml aqueous solution of $\mathrm{HAuCl}_{4}(0.001 \mathrm{M})$ and left for $1 \mathrm{~h}$ to impregnate. Afterward, AsA was added to the $5-\mathrm{ml}$ aqueous solution to achieve a $0.003 \mathrm{M}$ solution, and the solution was stirred for $10 \mathrm{~min}$. Finally, the membranes were rinsed with deionized water and sonicated for $10 \mathrm{~min}$ to remove any particles that were not within the PNIPAM grafts.

\section{Scanning electron microscopy (SEM)}

SEM images were obtained with aLEO 1530 scanning electron microscope (Oxford Instruments, Scotts Valley, CA, USA) at an acceleration voltage of $10 \mathrm{kV}$. The specimens were prepared by placing samples of the membranes on carbon-coated conducting tape and then coating the samples (unless specified otherwise as shown in each image) with silver, which allowed for better quality images and a higher resolution. Clean-cut images, which allowed analysis of the interior of the membrane, were obtained by breaking the membrane while it was submerged in liquid nitrogen. The membrane was soaked in water before submersion in liquid nitrogen.

\section{Atomic force microscopy (AFM)}

AFM images were acquired using an MFP-3D AFM (Asylum Research, Santa Barbara, CA, USA). Nonconducting silicon nitride-sharpened cantilevers (Veeco Metrology, Santa Barbara, CA, USA) were used for contact mode imaging.

\section{UV-vis spectroscopy}

UV spectra of the PC membranes with and without gold nanoparticle coupling were measured using a CARY $5000 \mathrm{UV}$-vis spectrophotometer (Varian Inc., Santa Clara, CA, USA)

\section{Heat conduction model of metal nanoparticles in the nanopores} of the polycarbonate membrane

The heat generated and conducted within the pores of the PCTPMs was modeled by analytically solving steady-state Fourier heat-transfer equations. The influence of the parameters, such as nanopore radius, size of the nanoparticles and laser spot diameter, were analyzed to determine the parameters needed to build optically responsive nanovalves.

The model neglects axial heat conduction in the nanopore. The validity of this approximation is analyzed in calculations included in the Supplementary Information.

\section{Thermal control of fluid flow}

Permeability changes in the membrane induced by temperature were determined by measuring water flow through the membranes. The membrane was taped to the end of a Pasteur pipette (diameter of $5 \mathrm{~mm}$ ) containing a water column of $5 \mathrm{~cm}(\sim 1 \mathrm{ml})$. Afterward, $0.43 \mathrm{ml}$ of water was allowed to flow through the membranes. The differential pressure was not kept constant in this experimental setup because the water column changed height as the flow passed. The respective times were recorded, and the average flow rate was determined. Additionally, the times for the sample membrane were normalized to an untreated PCTEPM for converting flow rates, using the Hagen-Poiseulle equation, to an effective pore size. Temperature-switching experiments of the membranes were performed by heating the volume of flowing water to three oscillations of temperature changes between $27^{\circ} \mathrm{C}$ and $40^{\circ} \mathrm{C}$.

\section{Optical control of fluid flow}

For the experiments to test water flow control in membranes using light, the same setup and procedure that was followed for testing thermal control of flow was followed. However, instead of temperature, light-switching experiments of the membranes were performed by switching the laser beam 'on' and 'off' at a wavelength of $530 \mathrm{~nm}$ at 2-min intervals. The flowing water was previously heated to $31{ }^{\circ} \mathrm{C}$, and the average water flow was recorded in response to the time that the membrane was exposed to the laser beam.

\section{RESULTS AND DISCUSSION}

The synthesis pathway developed to incorporate PNIPAM-metal nanocomposites into PCTEPMs involves two steps. Initially, PNIPAM chains are grafted to the surface of the PCTEPM through plasma initiation. The grafted membranes (gPCTEPMs) are then exposed to an aqueous solution of the metallic salt $\mathrm{HAuCl}_{4}$, which is reduced in situ using AsA. This ultimately achieves coupling of the polymer grafts to the metallic particles (Scheme 2).

\section{Synthesis pathway to incorporate PNIPAM into PCTEPMs}

A 'grafting from' technique was explored in this work to couple polymeric materials onto surfaces. This method has been shown to control the reaction and to obtain high densities of surface-grafted polymer. ${ }^{25}$ Several surface 'grafting from' techniques have been reported in the literature, including chemical grafting, ${ }^{25}$ plasma, ${ }^{24,26}$ radiation $^{13,19,27,28}$ and photoinduced grafting. ${ }^{29,30} \mathrm{~A}$ previously reported plasma-induced grafting technique ${ }^{24}$ was chosen for the grafting of PNIPAM to the PCTEPMs because it presented several advantages to incorporating the desired properties into the PC membrane. Plasma-induced grafting allows control over the location of the grafting of the PNIPAM chains, either on the membrane surface or on the walls of the pores. ${ }^{24}$ In addition, plasma treatment only affects the outer properties of the membrane, keeping its bulk properties unchanged. ${ }^{26}$

Plasma-induced grafting consists of exposing the PCTEPMs to an oxygen plasma cloud (air, in this case) to create metastable chemical radicals on the surface of the membrane that would eventually serve as initiators for the grafting polymerization reaction in the presence of the monomer (NIPAM). After plasma treatment but before initiation of the surface polymerization, the membrane was wetted with ethanol to promote grafting of the polymer in the pores. ${ }^{14}$ Afterward, the plasma-treated membranes were exposed to a $5 \mathrm{wt} \%$ NIPAM monomer solution at 30 and $20^{\circ} \mathrm{C}$ to initiate the surface polymerization. Thus, the polymer was finally grafted to the membrane surface at the top of the membrane and the walls of the pores, but not at the bottom where the plasma cloud could not reach. As a first experiment to test grafting of the polymer to the membranes, the membranes were glued to the tip of a Pasteur pipette, and a $5-\mathrm{cm}$ water column was added to the pipette. We observed that at room temperature, the membranes grafted at $20^{\circ} \mathrm{C}$ had considerable higher flows, when compared with the membranes grafted at $30^{\circ} \mathrm{C}$, where the water passage was basically blocked. This result showed that the $30^{\circ} \mathrm{C}$ grafting polymerization was more effective at covering the membrane pores; therefore, polymerization at $30{ }^{\circ} \mathrm{C}$ was the condition pursued further. 


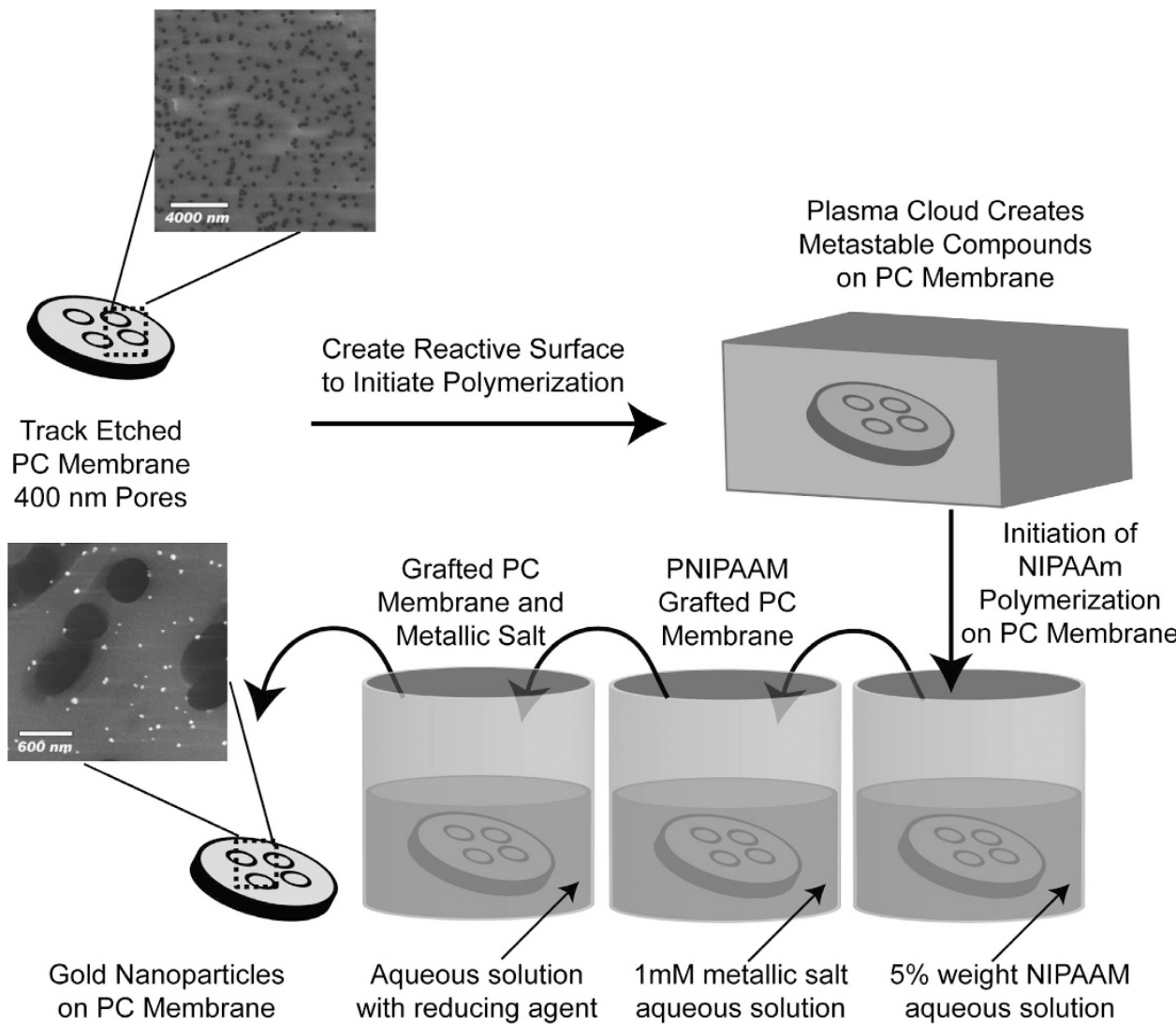

Scheme 2 NPCgPCTEPMs synthesis pathway. Initially, PNIPAM chains are grafted to the surface of the PCTEPM through plasma initiation. The grafted membranes (gPCTEPMs) are then exposed to an aqueous solution of the metallic salt $\mathrm{HAuCl}_{4}$, which is reduced in situ using AsA. This finally achieves coupling of the polymer grafts to the metallic particles.

The localization of the polymer grafts on each gPCTEPM sample was analyzed using SEM and AFM. Figures la and b shows the surface and interior pore structure, respectively, of the PCTEPM control. The surface of the gPCTEPM is shown in Figure 1c, and the interior structure of the pores can be observed in Figure 1d. The physical differences observed in the SEM images between the control PCTEPMs and the gPCTEPMs are very slight because both the PNIPAM grafts and the polycarbonate, of which the PCTEPMs are made, are amorphous polymers. Nonetheless, in the grafted membranes, the pores seem to have a thin film covering them. In addition, other slight differences, such as a loss of sharpness at the edges, can be observed between the samples (Figures 1a-d). Furthermore, analysis of the membranes with AFM allowed the visualization of the grafted PNIPAM in the PC membranes, as can be observed when comparing the PCTEPM to the gPCTEPM (Supplementary Figures S2a and b).

\section{Coupling nanoparticles to PNIPAM grafts within the porous membrane}

The coupling of metal particles onto the PNIPAM grafts in the gPCTEPMs is achieved through an in situ reduction of a metallic salt, $\mathrm{HAuCl}_{4}$, The membrane with the PNIPAM grafts was submerged in an aqueous solution of the metallic salt, followed by the addition of AsA, the reducing agent. After the addition of the reducing agent, the aqueous gold solution follows the typical color changes of a nanoparticle redox synthesis and proceeds until completion without the presence of a stabilizing and capping agent. The reaction initially becomes red rapidly and then quickly transitions into darker shades of blue. Finally, toward the end of the reaction, the particles form very large aggregates. However, when the gPCTEPMs are extracted from the reaction solution, the color of the membrane is a light ruby red; a color that is observed in gold nanoparticles of $10-50 \mathrm{~nm}$ diameters due to their surface plasmon resonance. ${ }^{23}$ We also note that the UV-vis spectra of the nanoparticle-coupled gPCTEPM, unlike the control gPCTEPM, shows a peak between 510 and $570 \mathrm{~nm}$, characteristic of gold nanoparticles ${ }^{23}$ (Supplementary Figure S1). This result demonstrates that the PNIPAM grafted in the porous membranes is acting as a stabilizing and capping agent in the synthesis of the metallic nanoparticles. This conclusion is strongly supported by our previous work, where a synthesis method was developed to control $\mathrm{Ag}$ and $\mathrm{Au}$ nanoparticle size and shape through the coupling of metallic nanoparticles to responsive polymers (PNIPAM). ${ }^{22,23}$ It was observed that the amine group in the PNIPAM ${ }^{22,23}$ and in other polymers $^{31}$ is capable of interacting with the metal, allowing for the stabilization of the nanoparticles, which achieves size and shape control.

The size and shape of the synthesized nanoparticles coupled to the polymer grafts were analyzed using SEM and AFM. As a control for the in situ reduction of the gold metallic salt, Figures $2 \mathrm{a}$ and $\mathrm{b}$ show the gold nanoparticles formed on the surface and pores of a PCTEPM, without PNPIAM grafts. Analysis of the membranes with AFM showed the grafted nanocomposite in the PC membranes, as can be observed when comparing the PCTEPM with the NP coupled gPCTEPM (Supplementary Figures S2a and c). Furthermore, it can be observed that most of the particles are aggregated and heterogeneously dispersed on the membrane. In addition, only a few particles are observed within the pores (Figure 2b). However, for membranes 

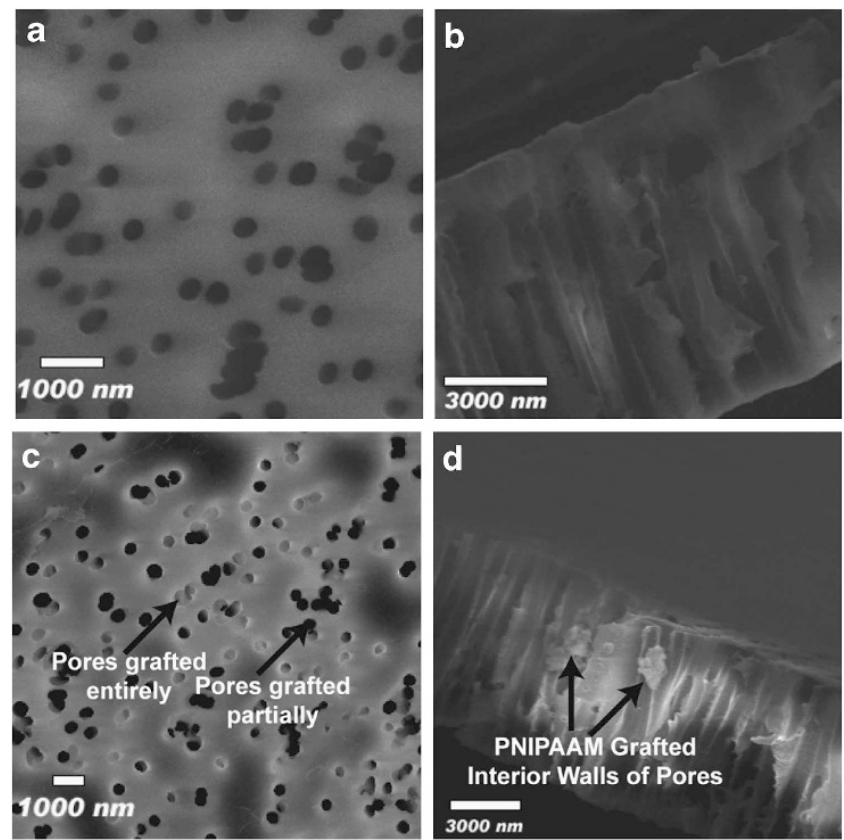

Figure 1 Poly( $N$-Isopropyl acrylamide) (PNIPAM) incorporated into polycarbonate track-etched porous membranes (PCTEPMs). (a-d) Scanning electron microscopy micrographs of the PCTEPMs. (a) Control PCTEPM surface without PNIPAM grafts. (b) Clean-cut internal structure of the control PCTEPM without PNIPAM grafts. (c) Surface and (d) internal structure of PCTEPM that has been grafted with PNIPAM.

that have been previously grafted with PNIPAM, the gold nanoparticles synthesized in situ have spherical shapes and are homogenously dispersed at the surface (Figure 2c) and the pores (Figure 2d) of the membrane. These results strongly suggest that the PNIPAM grafts are not only capable of controlling the synthesis of gold nanoparticles but also capable of coupling to them. Furthermore, Figure $2 \mathrm{~d}$ shows the presence of particles in gPCTEPM pores, which is also an indirect proof of the presence of PNIPAM grafts within the pores of the porous membranes.

The average diameter of the gold nanoparticles formed on the surface of the membranes is $42 \mathrm{~nm}$ (Figure 3a). This diameter is $60 \%$ larger than the average size previously reported for the synthesis in our previous work, which was carried out in a solution containing PNIPAM acting as a capping and stabilizing agent. ${ }^{23}$ Furthermore, the s.d. of the particles formed on the surface of the membranes grafted for the reaction is $\sim 40 \%$. When compared with the s.d. previously obtained for the reaction in solution, the s.d. of the present study is somewhat higher than when using high polymer-gold ratios $(22 \%$ achieved in solution) but similar to the 35\% obtained for low polymer-gold ratios. ${ }^{23}$ The higher s.d. an can be possibly attributed to impairment of PNIPAM chains to control size because they are grafted to a substrate with one end of the chains immobilized.

The size distribution of particles found on the surface (Figure 3a) was compared with that of the particles found within the pores of the samples (Figure 3b). From the data, two differences can be observed. First, the average size of the particles formed within the pores, $78 \mathrm{~nm}$ diameter, is $45 \%$ larger than the particles on the surface. Furthermore, the size distribution of the particles formed within the pores is $70 \%$ smaller than the particles formed on the surface of the membrane. These phenomena can be attributed to a diffusion effect within the nanopores that aids in the controlled synthesis of the nanoparticles.
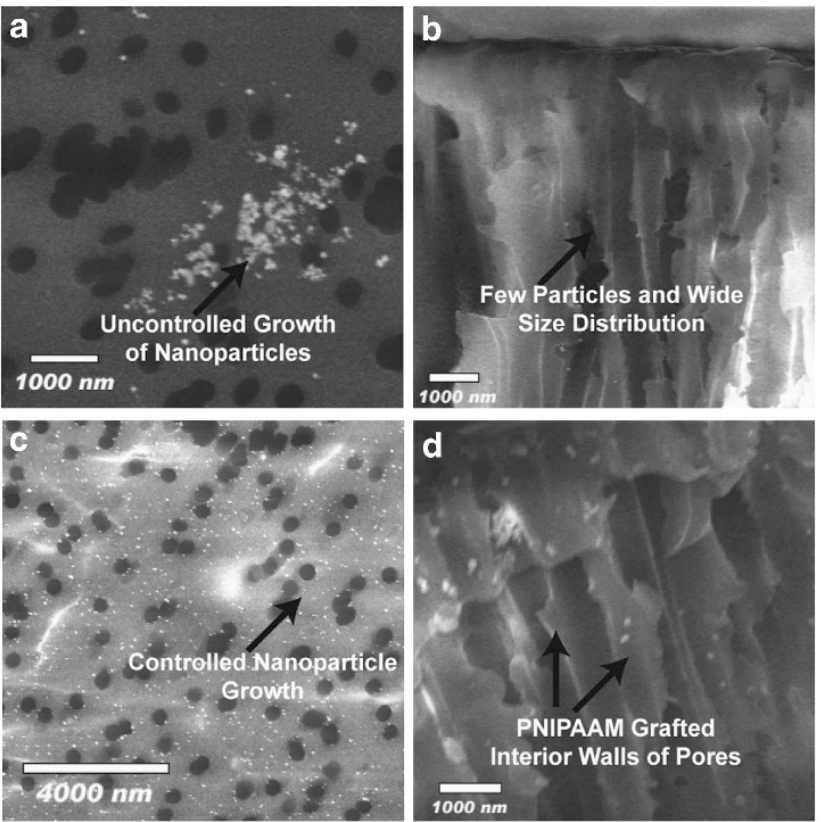

Figure 2 Metallic gold nanoparticles incorporated into grafted polycarbonate track-etched porous membranes (gPCTEPMs). (a-d) Scanning electron microscopy micrographs of the PCTEPMs. (a) Control PCTEPM surface without poly( $N$-isopropyl acrylamide) (PNIPAM) grafts after gold nanoparticles have been synthesized in situ. (b) Clean-cut internal structure of a. (c) Surface and (d) internal structure of PCTEPM that has been grafted with PNIPAM and gold nanoparticles have been synthesized in situ.
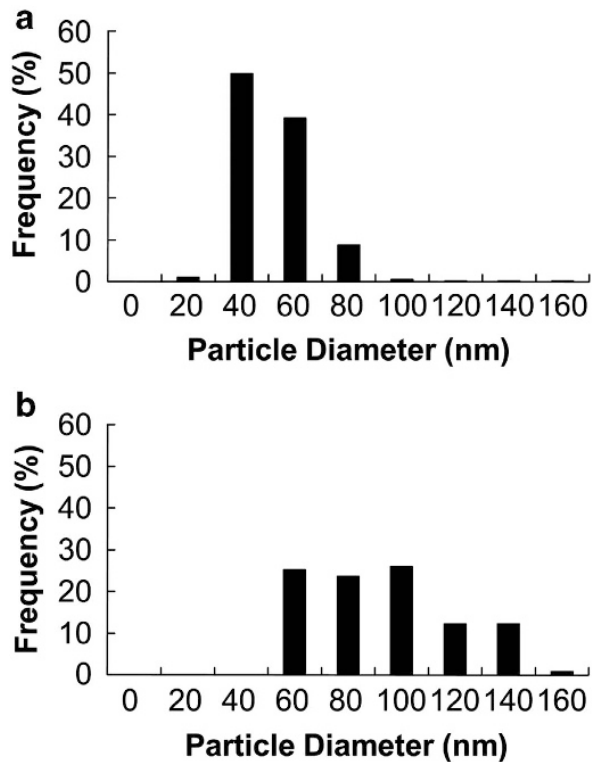

Figure 3 Size distributions of metallic gold nanoparticles incorporated into polycarbonate track-etched porous membranes (PCTEPMs). (a) Size distribution of the gold nanoparticles synthesized in situ on the surface of the gPCTEPM. The mean size is $42 \mathrm{~nm}$ diameter, with a s.d. of $40 \%$. (b) Size distribution of the gold nanoparticles synthesized in situ within the pores of the gPCTEPM. The mean size is $78 \mathrm{~nm}$ diameter, with a s.d. of $22 \%$. 


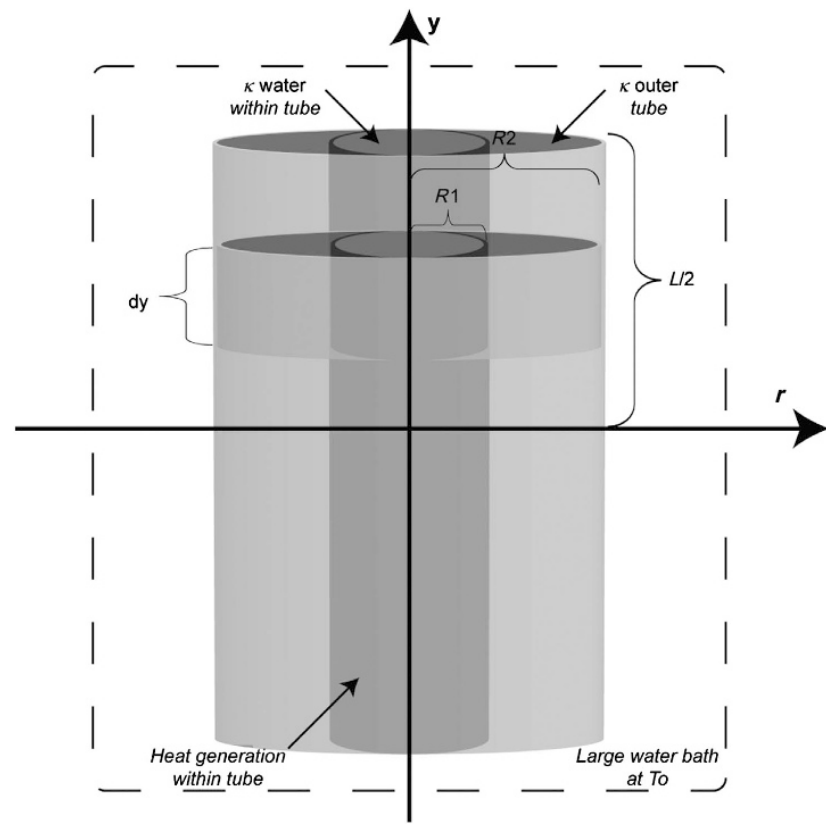

Scheme 3 Diagram of nanopore with heat generation to be mathematically modeled. $\kappa$ water is the heat conductivity of water, and $\kappa$ outer tube is the heat conductivity of polycarbonate. $R 1$ is the radius of the nanopore, and $R 2$ is the outer radius average distance between nanopores in the membrane. $L$ is the length of the nanopore, corresponding to the thickness of the membrane. It is assumed that the nanoparticles within the pore are homogenously dispersed. Therefore, when exposed to light, a homogenous heating of the interior of the pore will be induced.

\section{Theoretical model of the heat conduction exhibited in the nanopores of the PCTE porous membranes}

It was hypothesized that the optical switching of the nanoparticle polymer composite-grafted PCTEPM (NPCgPCTEPM) would be driven by the capability of metal nanoparticles to convert absorbed energy into heat. Therefore, an approach to model the heat generation and conduction within one of the nanopores of the NPCgPCTEPM was achieved by solving the Fourier's heat-transfer equation (all calculations are included in the Supplementary Methods section) for the system described in Scheme 3. The solution only included radial heat transfer and neglected the axial component of heat transfer. Demonstration that this is an accurate approximation is included in the Supplementary Methods. Modeling the system using the Fourier's heat-transfer equation determined the nanocomposite concentration and laser energy density needed to achieve enough heating in the local aqueous environment, a change of $1{ }^{\circ} \mathrm{C}$, which allows construction of the optical switching nanovalves proposed in this work. The simulation was performed using a nanoparticle with a 40-nm radius, as observed for the particle sizes achieved within the pores during synthesis, and with a cross-section calculated using a Mie coefficient of $1.8197 \times 10^{14} \mathrm{~m}^{2}{ }^{32}$ The outer radius of the pore used was $750 \mathrm{~nm}$ based on the pores of the control membranes in the SEM images of the control membranes and the average distance between the pores in the porous membranes. The rest of the parameters are listed in the Tables 1 . Solution of Fourier's equation allowed for calculation of (Supplementary Methods) the correlation between the volumetric heat generated and the change in temperature achieved in the nanopores (Figure 4a). Figure 4a shows a linear dependence between the number of particles within the pore and the heat generation achieved. It can be observed that $2 \times 10^{12} \mathrm{Wm}^{-3}$ of

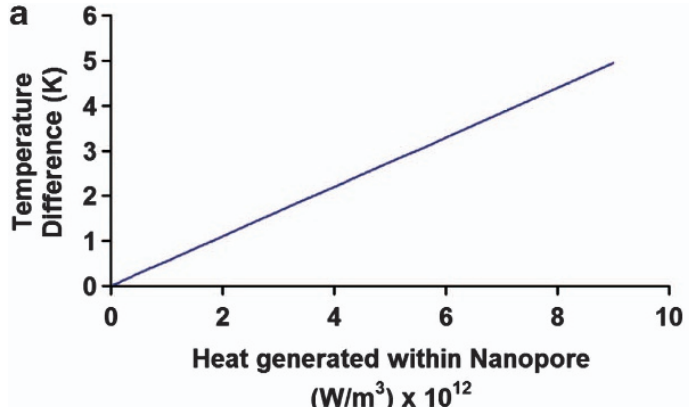

$\left(\mathrm{W} / \mathrm{m}^{3}\right) \times 10^{12}$

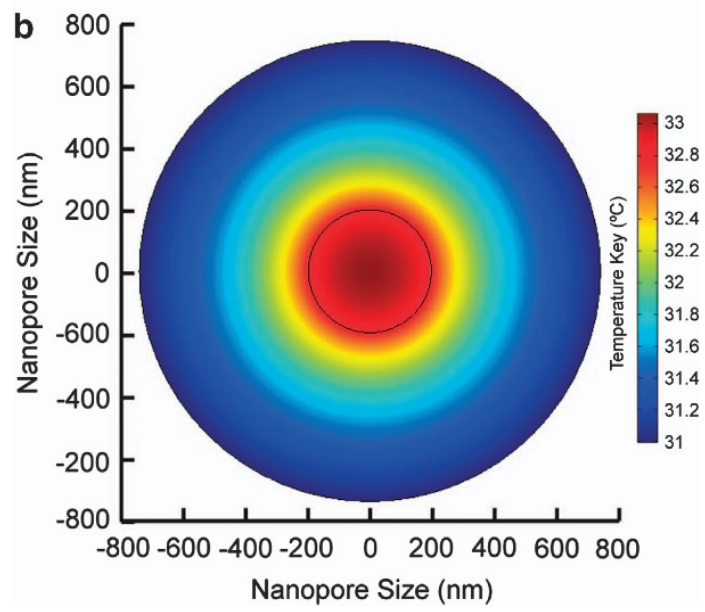

Figure 4 Theoretical heat generation and temperature distribution calculated for nanopores in nanoparticle polymer composite- grafted polycarbonate track-etched porous membranes (NPCgPCTEPMs). (a) Correlation between volumetric heat generation and temperature increment within the nanopore. (b) Steady-state temperature profiles in $400 \mathrm{~nm}$ diameter nanochannels. Simulation using FEMlab. Steady-state for the system is approached within less than a second.

Table 1 Parameters used to calculate heat generation within the polymer-metal nanoparticle colloid (nanoporous channel)

Parameters used in the mathematical model

Parameter

Value

Radius of the nanochannels

$200 \mathrm{~nm}$

Outer radius of the nanochannels

$750 \mathrm{~nm}$

Radius of the nanoparticles

$40 \mathrm{~nm}$

$\mathrm{Cp}$, specific heat of water

$4181 \mathrm{~J} \mathrm{~kg}^{-1} \mathrm{~K}^{-1}$

$\rho$, density of water

$\kappa_{\mathrm{W}}$, heat conductivity of water

$1000 \mathrm{~kg} \mathrm{~m}^{-3}$

$0.58 \mathrm{~W} \mathrm{~m}^{-1} \mathrm{~K}^{-1}$

$\kappa_{\mathrm{pc}}$, heat conductivity of polycarbonate membrane

$0.22 \mathrm{~W} \mathrm{~m}^{-1} \mathrm{~K}^{-1}$

$Q$, heat density generated within the colloid per time

$2.08 \times 10^{12} \mathrm{Wm}^{-3} \mathrm{~s}^{-1}$

heat generated within the pore is needed to achieve a $1-{ }^{\circ} \mathrm{C}$ difference in the entire pore and have a fast switching response because our previous work determined that these types of systems usually reach stationary phase within microseconds. ${ }^{23}$ The temperature profile within the channel is included, and it can be predicted, based on our previous work, ${ }^{23}$ that these temperature profiles will also be reached very rapidly (Figure $4 \mathrm{~b}$ ). Further calculations determined that the heat generation needed for switching can be achieved by focusing a $100-\mathrm{mW}$ power laser on a $40-\mu \mathrm{m}$ spot size radius into a volume with a concentration of $5.705 \times 10^{15}$ particles per liter. Using this 
a Thermal Switching Experiment
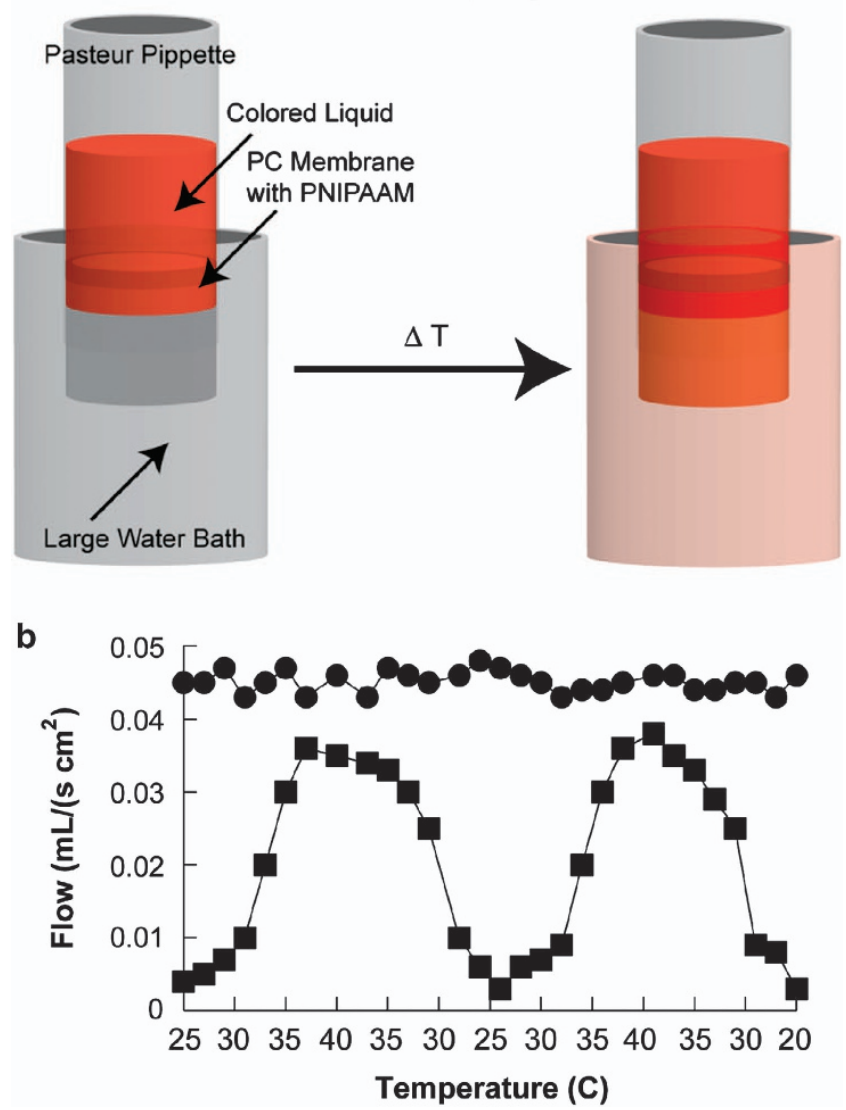

Figure 5 Reversible permeability switching of grafted polycarbonate tracketched porous membranes (gPCTEPMs) in response to heat. (a) Schematic of the experimental setup where the nanopores are switched from 'off' to 'on' using heat. (b) The flow changes shown correspond to the PCTEPMs without previous grafting (circles) and for gPCTEPMs (squares) in response to temperature.

concentration of particles and the known volume within the nanochannels, $8.796 \times 10^{8} \mathrm{~nm}^{3}$, the system predicts switching when approximately five particles can be localized within one channel. The synthesis developed in this work allows for the incorporation of a higher number of particles per pore of NPCgPCTEPMs than needed to achieve $1{ }^{\circ} \mathrm{C}$ heating. As a result, theoretical calculations predict that the nanoporous membranes engineered and built with the synthesis described in this work can most likely be used as optically switchable nanovalves when exposed to lasers of specific wavelengths and power densities.

Permeability switching experiments in response to heat

The PNIPAM chains grafted to the surface of the PCTE porous membranes, when exposed to heat, are expected to act as mechanical valves that control flow, as shown in Scheme $1 \mathrm{~b}$ and c. This test is required for verifying the grafting of polymeric materials onto polymeric membranes. Therefore, the ability to thermally and reversibly control flow through the pores in the membrane was tested in the syntheses where polymerization was conducted at 20 and $30^{\circ} \mathrm{C}$. Because the water column decreases as the water flows (Figure 5a), temperature-dependent average flows through the porous membranes were analyzed using gravity-driven flow with time-varying hydrostatic pressure starting at $500 \mathrm{~Pa}$ and ending at $200 \mathrm{~Pa}$. Fluid flow through the gPCTEPMs and NPCgPCTEPM was measured as a function of water temperature and plotted in Figure 5b. Several tests were performed on the switch and the control flow, and the results were reproducible, meaning the polymer and nanocomposites were stably grafted onto the membranes. Although there is low leakage of flow at low temperatures, when the polymeric nanocomposite is in its elongated conformation and is closing the pore (Scheme 1c), the PNIPAM gate can achieve nearly total blockage of the pores to convective flow. In addition, when the gate opens due to a change in conformation of the polymeric nanocomposite, flow changes from 0.001 to $0.035 \mathrm{ml} \mathrm{s}^{-1} \mathrm{~cm}^{-2}$ (Figure $5 \mathrm{~b}$ ) can be achieved, and the exact opposite effect is observed when the water goes from hotter to cooler temperatures. When the same experiments were conducted using the membranes polymerized at $20^{\circ} \mathrm{C}$, the flow changes ranged from $0.015-0.041 \mathrm{ml} \mathrm{s}^{-1} \mathrm{~cm}^{-2}$ (Supplementary Figure S3). This result suggests that the polymers were much shorter and proves that our synthesis method has the capability to control polymer length and molecular weight.

Studies with track-etched membranes often calculate effective pore diameters based on the Hagen-Poiseulle law for the flow rate in a cylinder: $V / \Delta t=$ Flowrate $=\pi \Delta P r^{4} / 8 \eta L$, where $V$ is the volume of the permeate, $\Delta P$ is the trans-membrane pressure, $r$ is the pore radius (assuming homogenous-sized pores in the membrane), $\eta$ is the viscosity of the permeate and $L$ is the capillary length (membrane thickness). The flow measurements in the present study were performed with time-varying pressure, and therefore, in the Hagen-Poiseulle equation, the time-averaged volumetric flow rate and pressure drop were used. The time-averaged pressure drop was constant in all the flow experiments. To exclude the viscosity changes with temperature and to be independent of the exact time dependence of the pressure decrease, all results are presented as relative to the control membrane. Therefore, the pore sizes were calculated using the following equation,

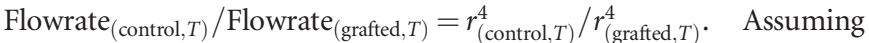
simple Hagen-Poiseulle flow, however, is inaccurate because the thickness of surface-grafted polymer layer in a cylindrical conduit is flow-rate dependent. Except for very low flow rates, we assume the polymer layer either swells due to shear ${ }^{33}$ or flattens if the order of the polymer brush is not very high and not all chains are pointing toward the flow (incomplete grafting). ${ }^{34,35}$ Additionally, if we assume that the polymer graft is a continuous gel with viscoelastic properties, which could be induced by embedding gold nanoparticles into the polymers, instabilities at the interface can develop even at very low Reynolds numbers. ${ }^{36,37}$

Despite the shortcomings of the Hagen-Poiseulle approximation, the pore size changes observed for the NPCgPCTEPMs show an effective change in pore diameters of $\sim 70 \mathrm{~nm}$, changing from an open pore in the collapsed state of $350 \mathrm{~nm}$ to a diameter of $280 \mathrm{~nm}$ in the hydrated state below the LCST.

The $\sim 70$-nm change in pore size between the collapsed and swollen state of the PNIPAM chains obtained in this work is similar to pore changes reported in the literature, which used the Hagen-Poiseulle equation and plasma-induced grafting. ${ }^{13,26,30,38}$ Switching responses with a bigger change in pore sizes, from 50 to $200 \mathrm{~nm}$, have been achieved using hydrogels; however, these systems present very long response times. ${ }^{39}$

Permeability switching experiments in response to light The experimental setup and pore size calculations (Hagen-Poiseulle) used for the thermal switching experiments were also used for 


\section{a Optical Switching Experiment}
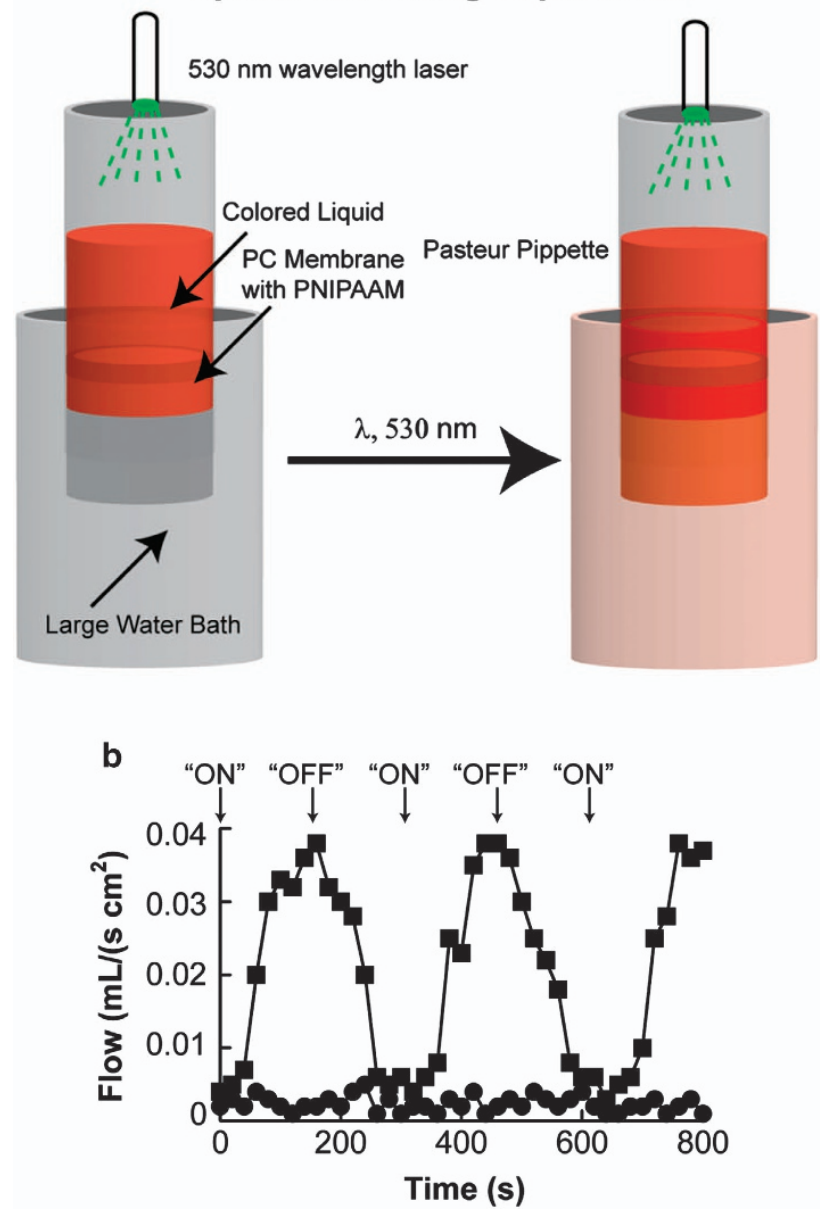

Figure 6 Reversible permeability switching of grafted polycarbonate tracketched porous membranes (gPCTEPMs) in response to light. (a) Schematic of experimental setup where the nanopores are switched from 'off' to 'on' using a nonfocused $530 \mathrm{~nm}$ laser. (b) The flow changes shown correspond to gPCTEPMs without coupled nanoparticles (circles) and with incorporated gold nanoparticles (squares) in response to light exposure times.

the light-induced permeability changes in the synthesized NPCgPCTEPMs (Figure 6a). The fluid flow through the gPCTEPMs and NPCgPCTEPM was plotted as a function of the time at which the membrane was exposed to the laser beams (Figure 6b). A behavior similar to that of the thermally induced reversible switching is observed, which is not at all surprising because the mechanical effect should be the same, but the actuator, light, is the only difference. Figure $6 \mathrm{~b}$ shows that flow control was very fast because fluid flow goes from 0.001 to $0.033 \mathrm{ml} \mathrm{s}^{-1} \mathrm{~cm}^{-2}$ in less than $2 \mathrm{~min}$. Furthermore, when the laser is shut off, a very similar opposite effect is observed. In addition, $50 \%$ of the increased total flow capability of the membrane is achieved within less than a minute of light exposure, which makes these membranes ideal for a wide range of applications.

In conclusion, PNIPAM grafting onto PCTEPMs was achieved by following the plasma-induced grafting techniques previously reported in the literature. ${ }^{24}$ Through an in situ reduction of metallic salts and by taking advantage of the capability of PNIPAM to act as a stabilizing and capping agent, PNIPAM grafts were coupled with gold metallic nanoparticles. Furthermore, size distributions and the shape of the synthesized nanoparticles were controlled by the PNIPAM grafts. In addition, the outcome of the synthesis described in this work suggested that within the pores, the diffusion effects allow for better control of nanoparticle sizes.

By using a mathematical model, this work predicts the simulated heat generation and conduction within a nanopore and suggests potential applications for the synthesized membranes as opto-thermally switchable valves when exposed to focused lasers of specific wavelengths and power densities. This work also demonstrates through permeability studies that reversible changes in flow can be achieved in the synthesized membranes through oscillatory changes in heat and light exposure.

\section{ACKNOWLEDGEMENTS}

The project was supported by Paicyt at the Universidad Autónoma de Nuevo León. We would also like to thank Dr Wolfgang Frey at the Department of Biomedical Engineering at the University of Texas for sharing his laboratory and for some of the chemicals used in this work.

1 Ma, W., Li, D. W., Sutherland, T. C., Li, Y., Long, Y. T. \& Chen, H. Y. Reversible redox of $\mathrm{NADH}$ and $\mathrm{NAD}(+)$ at a hybrid lipid bilayer membrane using ubiquinone. J. Am Chem. Soc. 133, 12366-12369 (2011).

2 Wang, H. Y., Ying, Y. L., Li, Y. \& Long, Y. T. Peering into biological nanopore: a practical technology to single-molecule analysis. Chem. Asian J./ 5, 1952-1961 (2010).

3 Branton, D., Deamer, D. W., Marziali, A., Bayley, H., Benner, S. A., Butler, T., Di Ventra, M., Garaj, S., Hibbs, A., Huang, X., Jovanovich, S. B., Krstic, P. S., Lindsay, S., Ling, X. S., Mastrangelo, C. H., Meller, A., Oliver, J. S., Pershin, Y. V., Ramsey, J. M., Riehn, R., Soni, G. V., Tabard-Cossa, V., Wanunu, M., Wiggin, M. \& Schloss, J. A. The potential and challenges of nanopore sequencing. Nat. Biotechnol. 26, 1146-1153 (2008).

4 Hou, X., Yang, F., Li, L., Song, Y. L., Jiang, L. \& Zhu, D. B. A biomimetic asymmetric responsive single nanochannel. J. Am. Chem. Soc. 132, 11736-11742 (2010).

5 Guo, W., Xia, H. W., Cao, L. X., Xia, F., Wang, S. T., Zhang, G. Z., Song, Y. L., Wang, Y. G., Jiang, L. \& Zhu, D. B. Integrating ionic gate and rectifier within one solid-state nanopore via modification with dual-responsive copolymer brushes. Adv. Funct. Mater 20, 3561-3567 (2010)

6 Zhang, L. X., Cai, S. L., Zheng, Y. B., Cao, X. H. \& Li, Y. Q. Smart homopolymer modification to single glass conical nanopore channels: dual-stimuli-actuated highly efficient ion gating. Adv. Funct. Mater. 21, 2103-2107 (2011).

7 Friebe, A. \& Ulbricht, M. Cylindrical pores responding to two different stimuli via surface-initiated atom transfer radical polymerization for synthesis of grafted diblock copolymers. Macromolecules 42, 1838-1848 (2009).

8 Kuo, T. -C., Cannon, D. M. Jr., Shannon, M. A., Bohn, P. W. \& Sweedler, J. V. Hybrid three-dimensional nanofluidic/microfluidic devices using molecular gates. Sensor. Actuat. A Phys. 102, 223-233 (2003).

9 Hediger, S., Fontannaz, J., Sayah, A., Hunziker, W. \& Gijs, M. A. M. Biosystem for the culture and characterization of epithelial cell tissues. Sensor. Actuat. B 63, 63-73 (2000)

$10 \mathrm{Hou}$, X., Zhang, H. C. \& Jiang, L. Building bio-inspired artificial functiona nanochannels: from symmetric to asymmetric modification. Angew Chem. Int. Ed. 51, 5296-5307 (2012)

11 Morones-Ramirez, J. R. Environmentally responsive polymeric "intelligent" materials: the ideal components of non-mechanical valves that control flow in microfluidic systems. Braz. J. Chem. Eng. 27, 1-14 (2010).

12 Hou, X., Guo, W. \& Jiang, L. Biomimetic smart nanopores and nanochannels. Chem. Soc. Rev. 40, 2385-2401 (2011).

13 Hautojarvi, J., Kontturi, K., Nasman, J. H., Svarfvar, B. L., Viinikka, P. \& Vuoristo, M. Characterization of graft-modified porous polymer membranes. Indus. Eng. Chem. Res. 35, 450-457 (1996).

14 Peng, T. \& Cheng, Y. -L. pH-Responsive permeability of PE-g-PMAA membranes. J. Appl. Polym. Sci. 76, 778-786 (1999).

15 Peng, T. \& Cheng, Y. -L. Temperature-responsive permeability of porous PNIPAAm-g-PE membranes. J. Appl. Polym. Sci. 70, 2133-2142 (1998).

16 Cartier, S., Horbett, T. A. \& Ratner, D. B. Glucose-sensitive membrane coated porous filters for control of hydraulic permeability and insulin delivery from a pressurized reservoir. J. Membr. Sci. 106, 17-24 (1995).

17 Kumar, S. K. \& Hong, J. -D. Photoresponsive ion gating function of an azobenzene polyelectrolyte multilayer spin-self-assembled on a nanoporous support. Langmuir 24, 4190-4193 (2008)

18 Brinker, C. J. Evaporation-induced self assembly: functional nanostructures made easy MRS Bull. 2004, 631-640 (2004).

19 Nayak, A., Liu, H. \& Belfort, G. An optically reversible switching membrane surface. Ang. Chem. Int. Ed. 45, 4094-4098 (2006). 
20 Lee, Y., Lee, H. S., Lee, J. S., Kim, S. K. \& Kim, S. H. Hormone- and light-regulated nucleocytoplasmic transport in plants: current status. J. Exp. Bot. 59, 3229-3245 (2008).

21 Wang, F. F., Lian, H. L., Kang, C. Y. \& Yang, H. Q. Phytochrome B is involved in mediating red light-induced stomatal opening in Arabidopsis thaliana. Mol. Plant 3, 246-259 (2010).

22 Morones, J. \& Frey, W. Environmentally sensitive silver nanoparticles of controlled size synthesized with PNIPAM as a nucleating and capping agent. Langmuir 23, 8180-8186 (2007).

23 Morones, J. R. \& Frey, W. Room temperature synthesis of an optically and thermally responsive hybrid PNIPAM-gold nanoparticle. J. Nanopart. Res. 12, 1401-1414 (2010).

24 Xie, R., Chu, L. -Y., Chen, W. -M., Xiao, W., Wang, H. -D. \& Qu, J. -B. Characterization of microstructure of poly( $\mathrm{N}$-isopropylacrylamide)-grafted polycarbonate track-etched membranes prepared by plasma-graft pore-filling polymerization. J. Membr. Sci $\mathbf{2 5 8}$ 157-166 (2005).

25 Alem, H., Duwez, A. -S., Lussis, P., Lipnik, P., Jonas, A. M. \& Demoustier-Champagne, $\mathrm{S}$. Microstructure and thermo-responsive behavior of poly(N-isopropylacrylamide) brushes grafted in nanopores of track-etched membranes. J. Membr. Sci 308, 75-86 (2008).

26 Huang, J., Wang, X., Chen, X. \& Yu, X. Temperature-sensitive membranes prepared by the plasma-induced graft polymerization of $\mathrm{N}$-isopropylacrylamide into porous polyethylene membranes. J. Appl. Polym. Sci. 89, 3180-3187 (2003).

27 Shtanko, N. I., Kabanov, V. Y., Apel, P. Y., Yoshida, M. \& Vilenskii, A. I. Preparation of permeability-controlled track membranes on the basis of 'smart' polymers. J. Membr. Sci. 179, 155-161 (2000).

28 Ulbricht, M. Photograft-polymer-modified microporous membranes with environmentsensitive permeabilities. React. Funct. Polym. 31, 165-177 (1996).

29 Yang, B. \& Yang, W. Thermo-sensitive switching membranes regulated by pore-covering polymer brushes. J. Membr. Sci. 218, 247-255 (2003).

30 Lequieu, W., Shtanko, N. I. \& Du Prez, F. E. Track etched membranes with thermoadjustable porosity and separation properties by surface immobilization of poly(Nvinylcaprolactam). J. Membr. Sci. 256, 64-71 (2005).
31 Sardar, R., Park, J. W. \& Shumaker-Parry, J. S. Polymer-induced synthesis of stable gold and silver nanoparticles and subsequent ligand exchange in water. Langmuir 23, 11883-11889 (2007).

32 Jain, P. K., Lee, K. S., El-Sayed, I. H. \& El-Sayed, M. A. Calculated absorption and scattering properties of gold nanoparticles of different size, shape, and composition: applications in biological imaging and biomedicine. J. Phys. Chem. B 110, 7238-7248 (2006)

33 Sevick, E. M. Shear swelling of polymer brushes grafted onto convex and concave surfaces. Macromolecules 29, 6952-6958 (1996).

34 Grest, G. S. Normal and shear forces between polymer brushes. Adv. Polym. Sci. 138, 149-183 (1999).

35 Chokshi, P. \& Kumaran, V. Stability of the flow of a viscoelastic fluid past a deformable surface in the low Reynolds number limit. Phys. Fluids 19, 104103-104103104103-104113 (2007).

36 Kumaran, V., Fredrickson, G. H. \& Pincus, P. Flow induced instability of the interface between a fluid and a gel at low Reynolds number. J. De Phys. I/ 4, 893-911 (1994).

37 Silberberg, A. Physico-chemical hydrodynamics in turbulent flows close to an interface. PhysicoChem. Hydrodyn. 9, 419-426 (1987)

38 Ulbricht, M., Ozdemir, S. \& Geismann, C. Functionalized track-etched membranes as versatile tool to investigate stimuli-responsive polymers for "smart" nano- and microsystems. Desalination 199, 150-152 (2006).

39 Lue, S. J., Hsu, J. -J., Chen, C. -H. \& Chen, B. -C. Thermally on-off switching membranes of poly( $\mathrm{N}$-isopropylacrylamide) immobilized in track-etched polycarbonate films. J. Membr. Sci. 301, 142-150 (2007).

(c) (1) (2) (2) This work is licensed under a Creative Commons
Attribution-NonCommercial-ShareAlike 3.0 Unported License. To view a copy of this license, visit http://creativecommons. org/licenses/by-nc-sa/3.0/

Supplementary Information accompanies the paper on the NPG Asia Materials website (http://www.nature.com/am) 